\section{Double trouble}

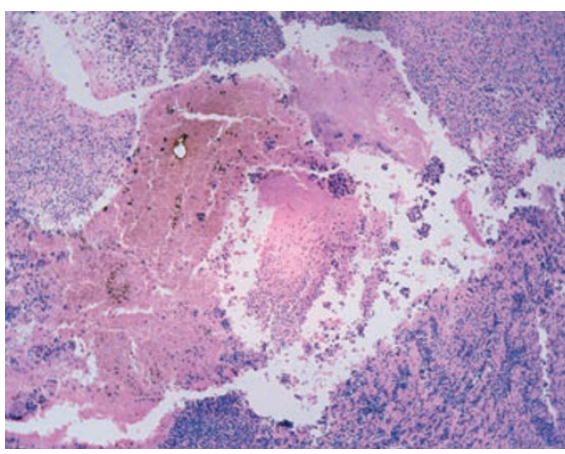

Chem. Commun. doi:10.1039/b713372c (2007)

Two species in the biochemical arsenal against cancer are nitric oxide (NO) and singlet oxygen $\left({ }^{1} \mathrm{O}_{2}\right)$. The correct dose of NO inhibits tumour proliferation and ${ }^{1} \mathrm{O}_{2}$ is the active species that causes cell death in photodynamic therapy. Now, a molecular system that simultaneously delivers $\mathrm{NO}$ and ${ }^{1} \mathrm{O}_{2}$ to the same region, under the control of visible light, has been reported by Salvatore Sortino and colleagues. The molecular assembly, which is about $40 \mathrm{~nm}$ in size, is a micellar aggregate of functionalized anionic porphyrin molecules and mesogen-containing cationic amphiphilic molecules. Within the aggregate, the two photoactive components are photochemically independent and, on irradiation with light, a supply of both $\mathrm{NO}$ and ${ }^{1} \mathrm{O}_{2}$ is emitted. The authors envisage that its small size, fluorescence and water-solubility make the nanoaggregate an appealing candidate for anticancer therapy in biological systems, and tests in this area are currently underway.

\section{Reactive oxidation}

J. Am. Chem. Soc. doi:10.1021/ja073712z (2007) Dimethyl ether (DME) can be produced either by direct conversion of synthesis gas or by dehydration of methanol. It is relatively non-toxic, inert and has similar characteristics to those of liquefied petroleum gas. As such it can be used as a convenient energy carrier for power generation and diesel replacement fuel, for example. Oxidation of DME on supported metal catalysts and homogeneous combustion pathways via radical intermediates have been investigated so far. Enrique Iglesia and colleagues now provide evidence for the identity and reversibility of the elementary steps required for catalytic combustion of DME on platinum clusters. Combining isotopic and kinetic analysis with theory, they demonstrated that reaction rates are limited by $\mathrm{C}-\mathrm{H}$ bond activation in DME molecules adsorbed on the surface. The combustion reaction was also affected by vacancies within chemisorbed oxygen layers and the

\section{9/11 reconstructed}

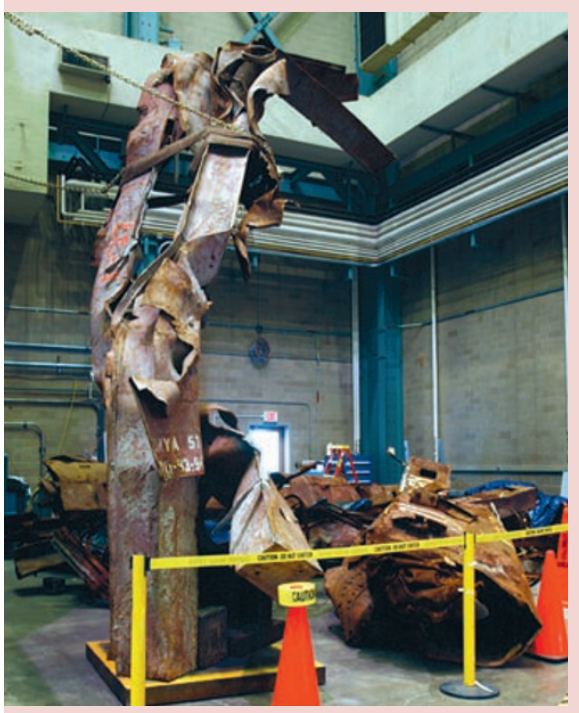

Mater. Res. Soc. Bull. 23, 710-716 (2007) Following the destruction of the World Trade Center on September 11, 2001, the US National Institute of Standards and Technology (NIST) painstakingly investigated the details of how the twin towers came to collapse. Their metallurgical findings have now been summarized for a broader audience by Frank Gayle, based on previously published detailed reports. To understand what led to the collapse, the NIST team first had to establish the mechanical properties of the 33 different types of steels used for the structural elements of the towers. These results were then fed into finiteelement models that simulated the impact of the aeroplanes. Further important clues were obtained from photographic evidence of the damage to some of the core columns inside the buildings. Importantly, in both towers it was the destruction of the fire protection of the steel columns that proved fatal. If that protection had remained intact, the twin towers would have withstood the attack. platinum cluster size. All in all these effects on reactivity should prove relevant to other oxidation reactions requiring vacancies on surfaces saturated with oxygen intermediates.

\section{What lies beneath}

Science 318, 76-80 (2007)

Polymers are often used as the insulating dielectric layers in organic thin-film transistors (OTFTs), but the glasstransition properties of such dielectric layers are rarely taken into account when considering the resulting OTFT performance. Tobin Marks and colleagues examine the correlation between the properties of dielectric polymers and the device characteristics of the pentacene OTFTs in which they are used. They find that the mobility of charge carriers in the devices drops by more than a factor of ten when the deposition temperature of the pentacene layer on top of the dielectric layer exceeds the surface - but not the bulk - glass-transition temperature of the polymer. The authors show that this change in mobility is a result of microstructural and morphological differences between pentacene grown on polymers in different viscoelastic states above and below the surface glasstransition temperature. They suggest that in future, transistor characteristics could be used to probe the properties of underlying polymers.

\section{Silicon does it alone}

Nature 449, 885-889 (2007)

Coaxial silicon nanowires may provide the basis for small solar cells that could power nanoelectronic systems. Nanorods and nanowires have previously been successfully used in attempts to reduce the size and improve the efficiency of solar cells. It has been suggested that coaxial structures could lead to further improvements, as the photogenerated carriers only need to cross the radial dimension of the wire, rather than travel in the longer axial direction. Charles Lieber and colleagues from Harvard University have now developed a coaxial wire based on a single material: silicon. Their structure comprises a p-doped core, with an undoped shell topped with an n-doped outer layer. These coaxial silicon wires have an energy-conversion efficiency of up to $3.4 \%$ and power output of up to $200 \mathrm{pW}$ per nanowire device - marked improvements on nanorod/polymer and nanorod/dye systems. 